\title{
Zur Geschichte der physiologischen Reizmethodik im 17. und 18. Jahrhundert*
}

\author{
Von K. E. Rothschuн, Münster (Westfalen)
}

\section{Einleitung}

Bei vielen physiologischen Experimenten bedient man sich heute ganz selbstverständlich einer wohldurchdachten Reiztechnik. Diese gibt uns die Möglichkeit, den Einsatz, die Dauer und die Stärke physiologischer Organaktivitäten planmäßig zu steuern und abzustufen. Das gilt vor allen Dingen beim Studium vom Herzen, vom Nerven- und vom Skelettmuskel, die wir durch Reizung vom Zustande der Ruhe in die Aktivität überführen wollen oder deren Aktivität wir in bestimmter Richtung verändern und beeinflussen wollen, ferner beim Gehirn, bei den Drüsen oder auch bei den Sinnesorganen.

Man versteht heute unter einem «Reiz» die auslösende Ursache für die Entfaltung und Abänderung der Eigenaktivität eines Organs (Rothschuh 1963, 295). Physiologische «Reize» können elektrischer, mechanischer, thermischer und aktinischer Art sein. Die Anwendung von Reizverfahren setzt voraus, daß Organe Reizbarkeit bzw. Erregbarkeit besitzen, die sich durch den Reiz wecken und verändern läßt.

Die Geschichte unserer Vorstellungen vom Reiz und von der Existenz einer Reizbarkeit lebendiger Teile zieht sich von der zweiten Hälfte des 17. Jahrhunderts bis ins 19. Jahrhundert hinein. Ihre Ideengeschichte sei hier nur am Rande behandelt, weil dieses Thema schon oft dargestellt worden ist (vgl. Verworn 1914, Buess 1942, Rothschuh 1958, Rudolph 1964). Doch steht diese Ideenentwicklung wieder im Zusammenhang mit der Ausgestaltung der Methodik des Reizens, also mit der Erfindung und Verbesserung von jenen auslösenden Eingriffen, die den Lebensvorgang provozieren oder beeinflussen können. Dazu ist aus historischer Sicht bisher wohl nur von G. Rudolph (1964) und D. Hüffmeier-von Hagen (1964) beigetragen worden. Zur Entfaltung dieser Reiztechnik im 17. und 18. Jahrhundert soll hier weiteres Material behandelt werden. Die Berichte über Reizversuche sind überall im Schrifttum der Zeit verstreut. Die folgende Zusammenstellung ist daher nicht mehr als ein erster Orientierungsversuch.

* Herrn Prof.Dr.ACKerKNEcht mit den herzlichsten Wünschen vom Verfasser. 


\section{Zufällige und unsystematische Reizversuche im 17.Jahrhundert}

Es ist natürlich altes Wissensgut, daß sich viele Lebensäußerungen des Menschen, z.B. der Puls, der Herzschlag, die Atmung, die Hautfarbe, die Harnabsonderung u.a.m., unter dem Einfluß diffuser Reize, z.B. von Wärme, Kälte, durch Essen und Trinken, Bewegung und Ruhe, Gemütsbewegungen usw., verändern. Auf diesen Erfahrungen beruht ja ein wesentlicher Teil der alten Therapie. Solange man ein solches Verhalten des Körpers nur im teleologischen Sinne als Reaktion einer zweckmäßig wirkenden Physis und des Gesamtorganismus interpretierte, stellte sich noch nicht die Frage, inwieweit auf jene Reize nur bestimmte Teile zwangsläufig und auf eigentümliche Weise reagieren. Ohne eine genauere Kenntnis der Organe, der Gewebe und ihres Zusammenhangs konnte weder die Idee einer besonderen Reizempfänglichkeit und einer Eigenaktivität der Teile unter dem Einfluß von Reizen Fuß fassen, noch ließ sie sich überprüfen oder beweisen. So ist es kaum verwunderlich, daß die Äußerungen einer besonderen Reizbarkeit erst bei vertiefter Beschäftigung mit der Anatomie der Teile, also erst seit dem 16. und 17.Jahrhundert, zur Beobachtung kamen. Ja, es mußte sogar erst an einzelnen Organen experimentiert werden, um zunächst zufällig und nachher planmäßig auf den Zusammenhang von Reizen mit bestimmten Organreaktionen zu stoßen. Das kam aber erst im 17. Jahrhundert im nennenswerten Umfang in Gang, als vom Tierversuch im Sinne des physiologischen Experimentes, also dem Versuch am lebenden Organismus oder überlebenden Teilen, stärker und stärker Gebrauch gemacht wurde.

Die Ergebnisse der ersten derartigen Reizversuche wurden auch durchweg mit großer Überraschung zur Kenntnis genommen. So schreibt R. LoWER $(1669)^{1}$ : «Welche gewaltige Veränderung des Herzens ergibt sich plötzlich, wenn die Nerven des 8. Paares fest abgebunden oder gänzlich durchschnitten werden ... dann beginnt das Herz ... zu klopfen und zu zittern ... auch erfolgt ein unmittelbares wildes Aufbäumen des Körpers beim Versuchstier» (mirum dictu quanta subito mutatio). Auch BELlin erzählte von dem überraschenden Ergebnis eines Versuchs: Als er bei geöffnetem Thorax den Zwerchfellnerv drückte, zog sich plötzlich das Zwerchfell zusammen $^{2}$. Das war nicht ganz leicht zu interpretieren. Da man im Nerv

1 Zit. nach der Ausgabe von D.Le Clerk und I. Jac. Mangetus 1685, S. 95.

2 Zit. nach Haller, Mémoires, Band I., S. 46, nach Liber de missione sanguinis... 
lediglich ein passives Gebilde zur Passage der Spiritus sah, kam gar nicht der Gedanke an eine Reizung und Eigenaktivität des Nervs auf, sondern man dachte nur an eine Unterbrechung des Zuflusses oder eine Verschiebung der Spiritus im Nerv.

Eine klarere Vorstellung vom Reiz hatte auch noch nicht Swammerdam, als er um 1658 seine Versuche am Nerv-Muskel-Präparat des Frosches ausführte (1738, II 835f.), um die Frage zu klären, ob der Muskel bei seiner Kontraktion an Volumen zunehme oder nicht. Dazu schloß er ein NervMuskel-Präparat, befestigt an einem Metallhaken ${ }^{3}$, in ein Glasrohr mit Steigröhrchen ein. Dann quetschte er den Nerv mit einem in diesen Versuchsraum eingeführten Silberdrähtchen mit dem Erfolg: «Nervus interpressus irritetur.» Auch er glaubte, mit dieser Quetschung den Nervenspiritus muskelwärts in Bewegung gesetzt zu haben. Übrigens liegt hier eine Kombination von mechanischer und elektrischer Reizanordnung vor. Allerdings dürfte die Reizwirkung der beiden Metalle, die im Spiele waren, effektlos geblieben sein, nur die mechanische Reizung dürfte nach der Schilderung gewirkt haben ${ }^{4}$. Swammerdam hat auch wohl als erster den Effekt der Nervendurchschneidung am Froschmuskel beschrieben ${ }^{5}$. Sodann hat Swammerdams Freund, Nikolaus Steno, nach seinem bekannten «Specimen » (1667) umfangreiche Versuche am Skelettmuskel und am Herzen ausgeführt. Er beschreibt z. B. die Zuckungen lebendfrischer Muskelfasern während ihrer präparatorischen Freilegung. Beim Durchschneiden der Vorderbeinnerven eines getöteten Hundes kontrahierten sich die Muskeln dieser Gliedmaße. Dasselbe sah er bei festem Druck auf einen Nerv. Steno behandelte das Thema erneut zusammenfassend im Jahre 1673. Er wendet bei Versuchen am Hund zur Weckung der Tätigkeit des ruhenden Herzens u. a. folgende Verfahren an: Er berührt das Herz mit dem Finger, er bläst es an, er sticht es oder reizt es mit seinem Messer. Das Kaninchenherz konnte er alleine durch seine Fingerwärme wieder zum Schlagen bringen, und das Herz eines frisch bebrüteten Hühnereis vom 7. Tag konnte er nach Herauspräparation auf die Hand nehmen und durch Anhauchen wieder zur Tätigkeit bringen: «Motum intermissum redire ad vellicationem sive a re solida, ut acu, cultro, ungue, sive a sanguine vel halitibus vel dilatatione»

3 «filum aereum».

4 Dibner 1952, S. 8, hat den Versuch u. a. elektrisch interpretiert.

5 «Dum cultello discinduntur aut punguntur nervi, motus exoriantur » Band I, S. 837. Man sollte, so meint der Autor später (S. 838), solche Versuche auch an Nieren, Leber, Milz und der Lunge versuchen. 
(editio 1685, 117). Er verwendete also vorwiegend mechanische und thermische Reize ohne Bemühung um eine Abstufung der Wirkungsstärke.

Das Herz ist ja überhaupt ein besonders geeignetes Organ, um auf dieses merkwürdige Phänomen der Reizbarkeit zu stoßen. Johannes Boнn beschreibt in seiner Jugendschrift Exercitationum Physiologicarum XXIII (1668) folgende Versuche, von denen der erste ein echter Reizversuch am Herzen ist: «Trenne aus dem Brustkorb eines lebenden Tieres das Herz heraus und beobachte es auf einem Tisch so lange, bis es völlig bewegungslos und ohne jedes Leben zu sein scheint; wenn Du dieses nun öfter mit der Spitze eines Messers oder einer Nadel berührst, wirst Du sehen, daß es sich bei jedem Stoß und bei der Wahrnehmung jedes Stoßes zusammenzieht; das ist ein handgreifliches Zeichen dafür, daß noch die Instrumente der Wahrnehmung vorhanden sind, nämlich die Spiritus animales, die nicht nur jede Belästigungen feststellen, sondern auch Gegenmaßnahmen treffen. Das heißt, sie sind fähig, jene Angriffe durch eine Konvulsion der Fasern abzuwehren.» Außerdem beschreibt Bohn, daß ein stillstehendes, erschlafftes Herz, wenn man es über ein Gefäß mit warmem Wasser aufhängt, durch diese Wärme wieder ins Leben zurückgerufen wird. Die Interpretation Bohns läßt aber erkennen, daß er auch hier nicht an eine unmittelbare Reaktion des Herzgewebes denkt, sondern an eine Verschiebung von Resten der Spiritus animales aus dem Herznerv in die Herzmuskelfasern.

Die Einsicht, daß die Kontraktion eines Muskels auf einer Eigenaktivität der einzelnen Muskelfasern beruht, wurde bekanntlich durch Fr. GLIsson angebahnt (1672, 1677). Es gibt nach ihm eine «motiva fibrarum facultas» oder die «Irritabilitas». Sie beruht auf einer constitutio insita der Faser. Ihre Kontraktion kann in dreierlei Formen auftreten: als ein motus naturalis (ohne Nerven- und ohne Gehirneinfluß), als ein motus sensitivus (unwillkürlich, aber über die Nerven) und als ein motus animalis (willkürlich durch einen influxus animalis). Aber nicht nur die Muskelfasern, sondern auch die übrigen Teile des Körpers sind irritabel, besonders auch das Herz gegenüber dem Blut. Doch haben die Organe eine verschiedene Abstufung ihrer Irritabilität (BERg 1942, RudolPH 1964). Es gehört zu jeder Faseraktion wie bei BoHN (siehe oben) eine «Perceptio », eine Empfindlichkeit, ferner ein «Appetitus», also die Fähigkeit, nach der Perceptio in Aktivität überzugehen. Die Perceptio ist natürlich keine bewußte Sensatio. GLIssons Vorstellungen waren noch recht spekulativ und nicht leicht verständlich, doch bahnten sie den Phänomenen der «Irritabilitas » den Weg.

Auch andere Forscher beschrieben gegen Ende des 17. Jahrhunderts ähnliche Beobachtungen, z. B. J. Duverney $(1700)^{6}$, Johann Gottfried v. Ber- 
ger (1701) und Georg Wolfgang Wedel (1704). Eine gewisse Bereicherung der Methodik der Muskelreizung gelang G.BAGLIvi. Dieser hat sich in vielen Schriften immer wieder mit der «Fibra motrice» und ihren Eigenschaften beschäftigt. So behandelt z. B. ein Kapitel des «Specimen de fibra motrice et morbosa » $(1701)^{7}$ die mannigfachen Wirkungen von Reizen. Baglivi beginnt mit der Darstellung klinischer Beobachtungen über Reizeffekte. Er meint, daß die Reizung der festen Teile des Körpers oft durch Beteiligung der Humores zustande komme, gewissermaßen durch eine humorale «Reizung»; denn wenn deren kleinste Teilchen allzu spitz oder salzig, erdig, stechend, scharf und pfefferartig sind oder wenn sie sich mit zu großer Geschwindigkeit bewegen, dann reizen sie die festen Teile, die in eine, sich u.U. ausbreitende, Oszillation geraten (editio 1827, 437). Vielleicht hat Baglivi aber auch noch durch andere Experimente Einfluß auf die Entwicklung der Reiztechnik genommen, denn bei der Präparation lebendfrischer oder Leichenmuskeln wandte er allerhand chemische Substanzen an, um durch Mazeration das Parenchym von den Fasern zu entfernen, z.B. Wein, Milch, Wasser, Alkohol, Essig, Sodawasser, Alaunwasser, Salmiakwasser, Sulfatlösungen, Scheidewasser, Königswasser und Laugen aus Pflanzenasche $(1715,266)^{8}$. Es ist zum mindesten wahrscheinlich, daß solche Versuche mit chemischen Stoffen oft Faserkontraktionen an den überlebenden Muskeln ausgelöst haben ${ }^{8}$. Jedenfalls wurden die chemischen Reize allmählich gebräuchlicher und vielseitiger. Ob post oder propter hoc, ist schwer zu sagen.

\section{Systematische Reizversuche im 18. Jahrhundert}

Baglivi glaubte bei diesen Präparationen zwei verschiedene Faserarten gefunden zu haben, nämlich die Fibra motrix und die Fibra membranacea. Die Fibra motrix verfügt über eine vis insita oder innata, also über eine angeborene, der Faser eigene Kraft der Zusammenziehung. Daß ein ausge-

${ }^{6}$ Es heißt dort: «Apparement il restoit encors dans ces nerfs de liqueurs, dont l'ondulation causoit le fremissement des parties ou ils répondoient et par consequent les nerfs ne seroient que des tuyaux, dont tout l'effect dépendroit de la liqueur qu'ils contiennent» (S.40).

7 Das Kapitel bei Baglivi ist überschrieben: «De irritatione solidorum, sive de stimulis, et de variis stimulorum effectibus», Liber II, Caput XI, Opera omnia.

8 «vino, lacte, aqua, spiritu vini, aceto, aqua nitrosa, aqua aluminosa, aqua armonniacalia, aqua vitriolica, aqua fortis, aqua regia, lixivo e cineribus.»

${ }^{8 a}$ Beobachtungen dieser Art beschreibt Baglivi in Opera omnia, 1827, S. 40. 
schnittenes Herz oder ein Muskel sich noch kontrahieren, beweist, daß nicht die Spiritus die wesentliche Ursache der Kontraktion sind, sondern diese vis insita. Natürlich hat Haller, der sich später $(1752 / 53)^{9}$ erneut mit der Unterscheidung von Fasertypen beschäftigt hat, Baglivis Schriften genau gekannt. Es ist aber überraschend, wie wenig er sich auf ihn bezieht. Das tut eingehender Hallers Schüler Johann Georg Zimmermann (1728 bis 1795) in seiner Dissertation von 1751, und zwar in der Einleitung. Diese Schrift ist zugleich die erste wesentliche physiologische Untersuchung mit systematisch angewandten Reizen und erweitert auch die bisherige Reizmethodik; denn Zimmermann verwendet schon eine ganze Reihe verschiedener Reizarten, besonders chemische und mechanische. Er prüft die Irritabilität der Organe chemisch besonders mit Vitriolöl und Antimonbutter ${ }^{10}$. Er reizt auch mechanisch durch Einschneiden mit dem Skalpell oder Stechen mit einem «filum acer». Auch erhitzt er das Skalpell und reizt damit thermisch (S. 21). Seine vielseitigen Ergebnisse können hier nur gestreift werden. Jedenfalls dürften diese Versuche das maßgebliche Ausgangsmaterial für die erwähnte Hallersche Abhandlung De partibus sensilibus et irritabilibus vom Jahre 1752/53 gewesen sein. Weiter beobachtete Zimmermann, daß ein gehirnloser Frosch durch eine Reizung seiner Pfoten heftig exzitiert wird (S. 29). Auch konnte dieser Frosch ohne Gehirn quaken (S. 34) ${ }^{11}$. Ein Stechen in das Rückenmark macht Konvulsionen, ein Druck auf den Zwerchfellnerv eine Bewegung des Zwerchfelles (S. 38). Warmes Wasser, Anhauchen, Einblasen von Luft durch die Vena cava (S. 58) genügen, um die Tätigkeit eines ruhenden, herausgenommenen Herzens anzuregen. Auch Eingeweide kontrahieren sich auf mechanische Reize, selbst nach dem Tode. Schließlich kommt Zimmermann zu der Überzeugung, daß die Irritabilitas eine allgemeine und wesentliche Eigenschaft bestimmter belebter Teile ist. «Irritabilitas est proprietas, quae in se contineat motus sui causam.» - «Si tolles irritabilitatem, tolleres vitam» (S. 70). Die Irritabilität stammt nicht von der Seele, nicht vom Blut und auch nicht vom Nerv, aber der Nerv trägt zum Grade der Irritabilität wesentlich bei (S. 65). Die Seele - das geht gegen StaHL - kann an diesen Erscheinungen nicht schuld sein, da ja diese Experimente an getöteten Tieren vorgenom-

9 Hallers Bericht wurde in Göttingen am 22. April und 6. Mai 1752 vorgetragen und erschien 1753 ebendort im Druck.

10 Antimonbutter ist eine alte Bezeichnung für Antimontrichlorid.

11 Man sieht, daß sowohl der «Anziehreflex» als auch der «Quakreflex» der Physiologie des 19. Jahrhunderts schon von ZIMMERMANN beobachtet wurden. 
men wurden und die Instrumente ohne Seele die Reaktionen in Gang setzten ${ }^{12}$. Leibniz glaube, daß den letzten Teilchen eine vis insita innewohne, aber das sei spekulativ. «Mihi videtor, irritabilitatem esse proprietatem corporum in primis animalium quae in se contineat motus sui causam » (70). Zimmermann erwähnt von Haller die Kommentare zu BoerhaAve, natürlich nicht dessen spätere Arbeit in den Göttinger Gelehrten Nachrichten (1752/53). Aber diese Hallersche Darstellung fußt im wesentlichen auf Zimmermanns Versuchen, faßt sie zusammen und diskutiert vor allem die klinische Seite. Allerdings gibt er dabei diesen Befunden eine differenziertere, wenn auch nicht besonders klare Deutung. Hallers Reizmethoden $(1752 / 53)$ unterscheiden sich in keiner Weise von denjenigen Zimmermanns. Mögen diese also tatsächlich von Zimmermann stammen, so wird doch wohl die Idee der systematischen Anwendung von Reizen von Haller selbst herrühren ${ }^{13}$ (vgl. auch Rudolph 1964, 19/20), denn er hatte schon vorher (1750), z.B. durch Foelix, eine Dissertation über die Bewegung der Eingeweide unter dem Einfluß verschiedener Reize ausführen lassen, und in der späteren französischen Ausgabe $(1756,116-399)$ ergänzt er den ersten Bericht durch die Aufzählung von 567 alten und neuen, nach 1752 angestellten Versuchen. Die Methoden ${ }^{14}$ sind die gleichen wie bei Zimmermann ${ }^{15}$. Haller berichtet auch in den Elementa (besonders in den Bänden I, II und IV) sehr ausführlich über die Wirkung von Reizen auf die verschiedensten Organe. Er reizt wieder mit Messer, Nadel, Dehnung, scharfen Salzen, Anhauchen, Wärme, Spießglanzbutter, Vitriol, Salmiakgeist-Spiritus. Auch der elektrische Reiz wird erwähnt. Besondere Reizkraft im Herzen entfaltet das Blut, auch das Hineinblasen von Luft. Die inneren Flächen der verschiedenen Abteilungen des Herzens sind augenscheinlich besonders und verschieden stark reizbar. Auch ist die Reizbarkeit der Herzen junger Tiere größer als diejenige erwachsener Tiere. Haller achtet also u.a. besonders

12 «in brutis jam emortuis mea instrumenta fecerunt» (S.65).

13 Tissot sagt in der Vorrede seiner französischen Übersetzung von HaLlers Versuchen (Mémoires) «c'est un unchainement de faits, qui ont été constatés, par une suite d'expériences faites avec la plus grande exactitude et réiterées très fréquemment pendant le cours de six ans, avant la publication de ce Mémoire» (Préliminaire, Band I, 1760, S.XXXV.

14 Z.B. avec le soufle, la chaleur, l'esprit de vin, le scalpel, le beurre d'antimoine, l'esprit de nitre, l'huile de vitriol, le canif, la pilore, le couteau; piquant de l'acier avec une éguille usw.

15 Die Frage des Anteils von Haller und Zimmermanv bedürfte einmal einer sorgfältigen Spezialuntersuchung. 
auf die unterschiedliche Reizbarkeit, wie sie sich bei den Versuchen ergibt, und zieht daraus wichtige Schlüsse. So ist um die Mitte des 18. Jahrhunderts nicht nur das Phänomen der Reizbarkeit bestimmter Organe, sondern auch die unterschiedliche Reizbarkeit qualitativ bekannt. Damit wollen wir uns aber hier nicht näher beschäftigen, sondern vielmehr verfolgen, wie sich die Reiztechnik in der zweiten Hälfte des 18. Jahrhunderts weiterentwickelte.

\section{Anfänge der elektrischen Reiztechnik im 18.Jahrhundert}

Über die Anfänge der Elektrophysiologie, auch der Methoden, wurde an anderer Stelle mehrfach berichtet (Rothschuh 1958, 1959, 1960). Ich kann mich daher hier kurz fassen. JALLABERT hat zuerst 1748 Muskelkontraktionen durch den Funken der Elektrisiermaschine auslösen können. Das setzte schon Apparate von erheblicher Leistungsfähigkeit voraus. Im Jahre 1756 hat zum ersten Male der AввÉ NoLLet den Schlag der Leydener Flasche auf den menschlichen Körper ausprobiert und gesehen, daß dabei heftige Schläge empfunden wurden. Haller erwähnt die Reizwirkung der Elektrizität ebenfalls, scheint sich aber selbst damit wenig beschäftigt zu haben. Im Anschluß an Hallers Hauptpublikation Von den empfindlichen und reizbaren Teilen des menschlichen Körpers entfaltet sich allerorts eine große reizphysiologische Aktivität ${ }^{16}$. Besonders interessant sind hier die Versuche, die von Caldani in Bologna ausgeführt worden sind. Er machte systematisch Reizversuche - meist mit mechanischen, gelegentlich mit elektrischen Reizen - an Froschmuskeln, aber auch an anderen Organen, um Hallers Versuche nachzuprüfen. Interessant ist auch, daß Fontana, der mit Caldani zusammenarbeitete, den elektrischen Reiz als die überlegene Methode (1757) bezeichnet ${ }^{17}$. Überhaupt war Fontana wohl der vorzüglichste Reizphysiologe des 18. Jahrhunderts. In seiner Schrift vom Jahre 1767, De Irritabilitatis Legibus, gelingt es ihm zum ersten Male, sehr klar die Existenz einer Phase der Reizunempfänglichkeit am Herzen festzustellen, also die «Refraktärphase» aufzufinden und richtig zu interpretieren ${ }^{18}$. Die Irritabili-

16 G. Rudolph (1964) hat die wichtigsten hierher gehörigen Publikationen in einem übersichtlichen Zeitdiagramm zusammengestellt.

17 Caldani berichtet u. a. (Hallers Mémoires $I I I, 1757 / 60$, §80, S. 126), wie er den elektrischen Apparat der Laura Bassi Veratti zu Reizversuchen benutzte und mit dem elektrischen Funken gearbeitet hat. FonTANA selbst (ebenda, Band III, S. 230) versuchte, die Herznerven (ohne Erfolg) mit dem elektrischen Funken der Elektrisiermaschine zu reizen. Beide Autoren wenden aber vorwiegend noch mechanische Reize an.

18 Vgl. dazu Marchand und Hoff (1955). 
tät ist nach ihm eine Eigenschaft, die nur den Fasern selbst zukommt und nichts mit den Spiritus zu tun hat. Bei seinen zahlreichen Versuchen, die er nicht im einzelnen beschreibt, sondern deren Ergebnisse er in einer sehr knappen und präzisen Sprache wiedergibt, verwendet er Stechen, Schlagen, Berühren mit einer Feder, Wassereinspritzungen und Blutfüllung beim Herzen. Er sieht dann z.B., daß Stiche mit einer feinen Nadel unmittelbar während der Herzkontraktion nicht wirksam sind, sondern erst nach einer gewissen Pause. Er findet den elektrischen Reiz mitunter wirksamer als die mechanischen Reize, die reiztechnisch weiter im Vordergrunde stehen. Die Spiritus, die ja dauernd zufließen, können gar nicht Ursache der wechselnden Herzkontraktion sein, denn ein andauernder mechanischer Reiz, z.B. durch eine eingestochene Nadel, macht nur eine einzelne Kontraktion, aber nicht eine wiederholte Tätigkeit. Überhaupt genügt die leichteste Berührung einer einzigen Faser des Herzens, um eine Kontraktion auszulösen. Froschmuskeln, die er mehrere Tage nach der Sektion reizte, kontrahierten sich noch und sicherlich ohne Anwesenheit von restlichem Spiritus animales. Diese Versuche hat Fontana später erweitert und darüber in einer weiteren Abhandlung 1775 berichtet (vgl. Übersetzung von Hebenstreit 1785). An kopflosen Fröschen hat er auch das Rückenmark elektrisch gereizt. Bei längerer Reizung wurden die Tiere schließlich unbeweglich und reagierten nicht mehr auf einen zusätzlichen Einzelreiz. Die stark mit Wasser gefüllte und gedehnte Harnblase verliert ihre Irritabilität gegen Nadelstiche und Elektrizität. Ähnlich verhält sich das Herz. Druck, Zug und Kälte können die Irritabilität vernichten. Fontanas Versuche sichern also über Haller hinaus das Phänomen vorübergehender Reiz-Unempfänglichkeit und die große Rolle, die gewisse Bedingungen und Umstände für die Abstufung der Reizbarkeit haben, sei es nun am Herzen in der Folge von Kontraktion, Erschlaffung und U̇berdehnung oder an Skelettmuskeln nach Vorbehandlung mit verschiedenen Mitteln. Die quantitative Stärkenabstufung der Reize steht noch ganz in den Anfängen. Dieses alles spielt sich noch in der vorgalvanischen Ära ab.

\section{Reiztechnik in der Zeit des Galvanismus}

Luigi Galvani, Schüler von Caldani in Bologna, hatte bekanntlich am Froschschenkelpräparat seit 1780 eine Unzahl von elektrischen Reizversuchen gemacht, zunächst mit der Elektrisiermaschine und der Leydener Flasche (vgl. Sirol 1939). Dann kam er zur Anwendung von verschiedenen Metallkombinationen und beobachtete mit dieser «Armatur» ein Zucken des Präparates. GaLvani interpretierte diese Beobachtung anfänglich als Metall- 
elektrizität, später als Folge der Entladung einer eigenen «tierischen » Elektrizität. VoLTA widersprach nach anfänglicher Zustimmung dieser Auffassung, und in der Auseinandersetzung zwischen Galvani und Volta, die sich bis zum Tode Galvanis im Jahre 1798 erstreckte, wurden die Reizanordnungen in zahlreicher Hinsicht variiert und vervielfältigt, wobei schließlich von GALVANI auch Versuchsanordnungen gefunden wurden, bei denen auch ohne Metalle Reizwirkungen erzielt wurden.

Galvanis Leistung im Zusammenhang mit der Reiztechnik beruht also darauf, daß er mit seiner Metallarmatur (d.h. einer Verbindung von zwei verschiedenen Metallen und einem flüssigen Leiter) ein vollkommen neues und sehr einfaches Reizverfahren fand. Damit ist in den nächsten Jahren, vor allen Dingen zwischen 1792 und 1800, sehr viel gearbeitet worden. Daran waren außer Galvani und Volta (vgl. Rothschuh 1964) besonders beteiligt Carl Créve (1793), Christoph Heinrigh Pfaff (1795), Alexander von Humboldt (1797) und Johann Wilhelm Ritter (1797, 1798). Die Versuche von Pfaff (1795) wurden mit ganz besonderer Beobachtungs- und Experimentierkunst durchgeführt. Er prüfte u.a. die Wirkung verschiedener Luftarten auf die Reizbarkeit des galvanischen Präparates und probierte auch die verschiedensten Metalle in ihrer abgestuften Reizwirkung aus. Auch Humboldt hat viele Jahre hindurch unermüdlich Reizversuche ausgeführt. Er behauptet in seinem Buch über die gereizte Muskel- und Nervenfaser, daß er an rund 3000 Tieren experimentiert habe. Humboldts Experimentierkunst war allerdings sehr wenig systematisch (vgl. Rothschuh 1959). Er verwendete nicht nur die verschiedensten Metalle, sondern auch seltsame Kombinationen von Leitern und Nichtleitern, Metallen und Salzen, Seifen und Ölen, von Fetten und Kohle. Trotz unzähliger Versuche gelang es ihm nicht, zu klaren Schlüssen zu kommen.

Zur Reiztechnik dieser galvanischen Ära ist zu sagen, daß man vorwiegend mit den erwähnten Metallarmaturen experimentierte. Diese lieferten allerdings nur sehr kleine Spannungen (etwa 1 Volt) und bei den hohen Widerständen der Präparate auch nur geringe Stromstärken. Sie waren also in der Intensität der Reizwirkung den alten elektrischen Anordnungen, wie der Leydener Flasche und der Elektrisiermaschine, mit ihren hohen Spannungen eher unterlegen. Deswegen ließen die galvanischen Beobachtungen auch zunächst keine Anzeichen einer Polarität erkennen. Auch war die Reizwirkung der Metallarmaturen auf die Skelettmuskelfaser und den Herzmuskel recht unzuverlässig. Nur wenn ein Nerv in die Anordnung einbezogen war, so waren die Ergebnisse einigermaßen konstant. Erst als im Jahre 1800 Alessandro Volta die Voltasche Säule erfand, hatte man bei mittel- 
hohen Spannungen (je nach der Anzahl der Plattenpaare bis zu 200 Volt) relativ große Stromstärken zur Verfügung. Von jetzt an war eine quantitative Abstufung der Reize möglich, damit wurde die Reiztechnik differenzierter und wurden die Ergebnisse eindeutiger. Immerhin haben schon vorher Fontana, Pfaff, Humboldt und Ritter gute Versuche über die qualitative Beeinflussung der Irritabilität ausgeführt, z. B. unter Alkohol, Opium-Alkohol-Lösung, Alkalien, Salpeter, Schwefel, Moschus und Campher.

Ganz entscheidende Verdienste um die Weiterentwicklung der Reiztechnik erwarb sich zweifellos Johann Wilhelm Ritter (1776-1810). Einschlägiges findet sich sowohl in seinem ersten Buch Beweis, das ein beständiger Galvanismus den Lebensprozeß im Thierreich begleite (1798), vor allen Dingen aber in seiner späteren Schrift Beyträge zur näheren Kenntnis des Galvanismus und der Resultate seiner Untersuchung (1801-1805) (vgl. Hüffmeier 1964).

Ritter verwandte ausgiebig von ihm selbst zusammengestellte stärkere Reizquellen, vor allen Dingen aber auch die Voltasche Säule; er hatte damit relativ starke elektrische Strom- und Spannungsquellen zur Verfügung. Es gelang ihm damit erstmalig, die polaren Effekte bei Reizung der Zunge, des Auges, des Gehörorgans festzustellen. Er sah also, daß der eine und der andere Pol durchaus verschiedenartig wirken kann. Ritter hat auch zum ersten Male die «einschleichende» Reizung ${ }^{19}$ beschrieben, er ließ den Strom langsam stärker werden, und dabei hatte der Strom trotz relativ großer Stärke keinerlei Wirkung.

Ritter zeigte ferner, daß während der konstanten Durchströmung bei geschlossener galvanischer Kette Änderungen der Erregbarkeit des Nervs an den beiden Polen auftreten (= späterer «Elektrotonus») ${ }^{20}$. Er sah SchlieBungs- und Öffnungszuckungen je nach Polung und Stromstärke getrennt voneinander auftreten. Ritter konnte auch bestätigen, daß die Erregbarkeit eines Nervs durch Chemikalien herabgesetzt wird, und beschrieb am absterbenden Nerv verschiedene Erregbarkeitsvariationen. Er stellte auch ein Zuckungsgesetz auf, das in den Grundzügen dem späteren «Pflügerschen Zuckungsgesetz» entsprach.

Besonders mutig und aufopfernd waren die Selbstversuche Ritters, als er Auge und $\mathrm{Ohr}$ mit starken Strömen zu reizen versuchte. Dabei gelang es ihm z.B., bei einer elektrischen Reizung seiner Ohren mit der Voltaschen

19 Besonders in 1805, II 2, S. $44 \mathrm{ff}$.

20 Weiteres findet man bei D. HüFfmeIER-von Hagen (1964). 
Säule deutliche Töne zu erzeugen, die er in Beziehung zur Stromstärke setzen konnte. Auch gab er an, daß der Ton nicht nur bei Schließung, sondern auch bei konstanter Durchströmung der Kette zu hören sei. Ähnliche Versuche machte er auch am Auge. Ritters Beobachtungen waren von großer Systematik und Folgerichtigkeit, ganz im Unterschied zu denen von Alexander von Humboldt. Die polaren Effekte, die Ritter beobachtete, wurden zwar für die romantische Naturphilosophie der Ausgangspunkt umfassender Spekulationen, waren aber auch der Ausgangspunkt der Elektrochemie.

Damit findet - jedenfalls in Deutschland -für eine Weile die Fortentwicklung der Reizmethoden ein Ende. Erst in den vierziger Jahren des 19. Jahrhunderts beginnt das ganze Gebiet neues Interesse zu finden. Um seine Wiederbelebung hat sich neben Carlo Matteucci (1811-1866) vor allen Dingen Emil du Bors-Reymond (1818-1896) verdient gemacht. Mit dieser Entwicklung hebt die moderne Phase der Reiztechnik an, in der man schließlich ganz zu elektrischen Verfahren übergegangen ist.

Das Ergebnis dieser mehr als 150 Jahre dauernden Zeitspanne, in der man sich zunächst mit unsystematischen und später mit systematischen Reizversuchen um die Identifizierung und die Klärung der besonderen Eigenschaften reizbarer Organe bemühte, war eine mehr oder minder klare Vorstellung über die Natur der Reize, das Phänomen der Erregung und die Erregbarkeit, über die Reizschwelle und die Refraktärphase. Von den mechanischen Reizen ausgehend, gelangte man über Wärmereize, Flüssigkeits-, Luft- und Blutreize zu chemischen Reizen und schließlich zu wirksamen und quantitativ nach Dauer und Intensität abstufbaren elektrischen Reizen.

\section{Schrifttum}

Aldini Jean (1804), Essai théorique et expérimentale sur le galvanisme, Paris.

Baglivi Giongio (1701), Specimen de fibra motrice et morbosa, Rom; Opera omnia, I., Leipzig 1827, S. $433 \mathrm{ff}$.

- (1715), De fibra motrice, in Opera omnia medicopractica et anatomica, Antwerpen.

Berg Alexander (1942), Die Lehre von der Faser als Form- und Funktionselement des Organismus, Virchows Arch.309 (1942) 333-460.

Berger Johannes Gottfried (1701), Physiologia Medica, Vitembergae.

Вонм Joн., Circulus Anatomico-Physiologicus (Oeconomia corporis animalis), Leipzig 1686, S. 97-98.

- Exercitationum Physiologicarum XXIII, Lipsiae 1668 (besonders Diss. IX., § 10/2).

Brazier M. A., Felice Fontana in Essays on the. History of Italian Neurology, ed. Luigi Belloni, Milano 1963, S.107ff. 
Buess Heinrich (1942), Zur Entwicklung der Irritabilitätslehre, Festschrift Dr.BrodbeckSandreuter, Basel, S. $299 \mathrm{ff}$.

Caldani M.A. (1757), Lettre I (1756) und Lettre II in A.von Hallers Mémoires sur la nature sensible, Band III, Lausanne 1760, S. 345.

Créve Carl Caspar (1793), Beiträge zu Galvanis Versuche (!) über die Kräfte der thierischen Elektrizität auf die Bewegung der Muskeln, Frankfurt/Leipzig.

Dibner Bernd (1952), Galvani-Volta, Norwalk (Conn.).

Duverney J. (1700), in Histoire de l'Academie Royale des Sciences, zweite Ausgabe, Paris 1719, Mem.XII, S. 40.

Foelix Jacobus (1750), Diss. De motu peristaltico, Göttingen.

Fontana Felice, Beobachtungen und Versuche über die Natur der thierischen Körper, Übersetzung von E.B.C. Hebenstreit, Leipzig 1785.

Galvani Aloysius, De viribus electricitatis in motu musculari commentarius, Mutina 1792; deutsch in Ostwalds Klassiker der exakten Wissenschaften, Nr.52, Leipzig 1894.

GuIsson Fr. (1672), Tractatus de natura substantiae energetica..., London.

- (1677), Tractatus de ventriculo et intestinis, London (besonders 7.Kapitel).

Haller A.v. (1752/53), De partibus corporis humani sensilibus et irritabilibus, Comm. Soc. Reg.Sci., Göttingen, ad Annum 1752, Band II, S.114-158, Göttingen 1753.

- Mémoires sur la nature sensible et irritable des parties du corps animal, Band I, Lausanne 1756; Bände II-IV, 1760.

- Anfangsgründe der Physiologie des menschlichen Körpers, aus dem Lateinischen übersetzt von Joh. Samuel Haller, Band I, Berlin 1759.

- Elementa Physiologiae, besonders Band II, 1760, S. 305.

HüFFMeier D. -von HAGEN (1964), J. W. Ritter und die Anfänge der Elektrophysiologie in Von Boerhaave bis Berger, herausgegeben von K. Е. RотнеснUн, Stuttgart, S.48-61.

Humboldt Alexander von (1797), Versuche über die gereizte Muskelfaser nebst Vermutungen über den chemischen Proze $\beta$ des Lebens in der Thier- und Pflanzenwelt, Band I und II; Posen/Berlin.

JaLlabert JeAn, Expériences sur l'électricité avec quelques conjectures sur la cause de ses effets, Genève 1748, Paris 1749.

Lower Rich., Tractatus de Corde, zit. nach Bibl.Anatomica, ed.D. Le Clerk und I. JAC. MANGetus, Band II, Genève 1685.

Marchand J.F. und H.E.Hoff (1955), Felice Fontana: The Laws of Irritability, J. Hist. Med.10 (1955) 197-206, 302-326, 399-420.

Pfaff Chr. H. (1795), Über thierische Elektrizität und Reizbarkeit, Leipzig.

Ritter Јон. WiLH. (1798), Beweis, daß ein beständiger Galvanismus den Lebensprozeß im Tierreich begleite, Weimar.

- Beyträge zur näheren Kenntnis des Galvanismus und der Resultate seiner Untersuchung, Jena, Band I 1801, Band II 1805.

Rотныснин K.E. (1953), Geschichte der Physiologie, Heidelberg.

- (1958), Vom Spiritus animalis zum Nervenaktionsstrom, Ciba-Z.8 (1958) 2950.

- (1959), Aus der Frühzeit der Elektrobiologie, Elektromedizin 4 (1959) 201.

- (1959), Alexander von Humboldt und die Physiologie seiner Zeit, Sudhoffs Arch.Gesch. Med. Naturwiss. 43 (1959) 97-113. 
- (1960), Von der Idee bis zum Nachweis der tierischen Elektrizität, Sudhoffs Arch. Gesch.Med. Naturwiss.44 (1960) 25-44.

- (1963), Die neurophysiologischen Beiträge von Galvani und Volta, in Essays on the History of Italian Neurology, ed.Luigi BeLloni, Milano, S. $117 \mathrm{ff}$.

- (1963), Theorie des Organismus, München.

- (1964), Jean Riolan jun. (1560-1657) im Streit mit Paul Marquart Schlegel (1605-1653) um die Blutbewegungslehre Harveys, Gesnerus 21 (1964) 72-82.

Rudolph G. (1964), Hallers Lehre von der Irritabilität und Sensibilität in Von Boerhaave bis Berger, herausgegeben von K. E. RотнSснUн, Stuttgart, S.14ff.

Sirol Marc (1939), Galvani et le Galvanisme, L'électricité animale, Thèse Médicale Toulouse, Paris.

Steno Nicolaus, Ex variorum animalium sectionibus hinc inde factis excerptae observationes circa motum cordis auricularumque et venae cavae, Acta Hafniensa I., Hafniae 1673, Obs. 46 und zit. nach Bibliotheca Anatomica, herausgegeben von D. LE CLERK und I. Jac. Mangetus, Band II, Genevae 1685, S.95.

Swammerdam Joannis (1738), Biblia Naturae sive Historia Insectorum. Herausgegeben von H.BoerhaAve und H.D.Gaubius, Leydae (besonders Band II, Tab. XLIX, Text S. 835-860).

Verworn Max (1914), Erregung und Lähmung. Eine allgemeine Physiologie der Reizwirkungen, Jena.

Wedel Georg Wolfgang (1704), Physiologia Medica, quattuor Sectionibus destinata, ed. sec., Jenae 1704.

Zimmermann Joannes Georgius (1751), Diss.physiologica de irritabilitate, Göttingen, 75 Seiten. 


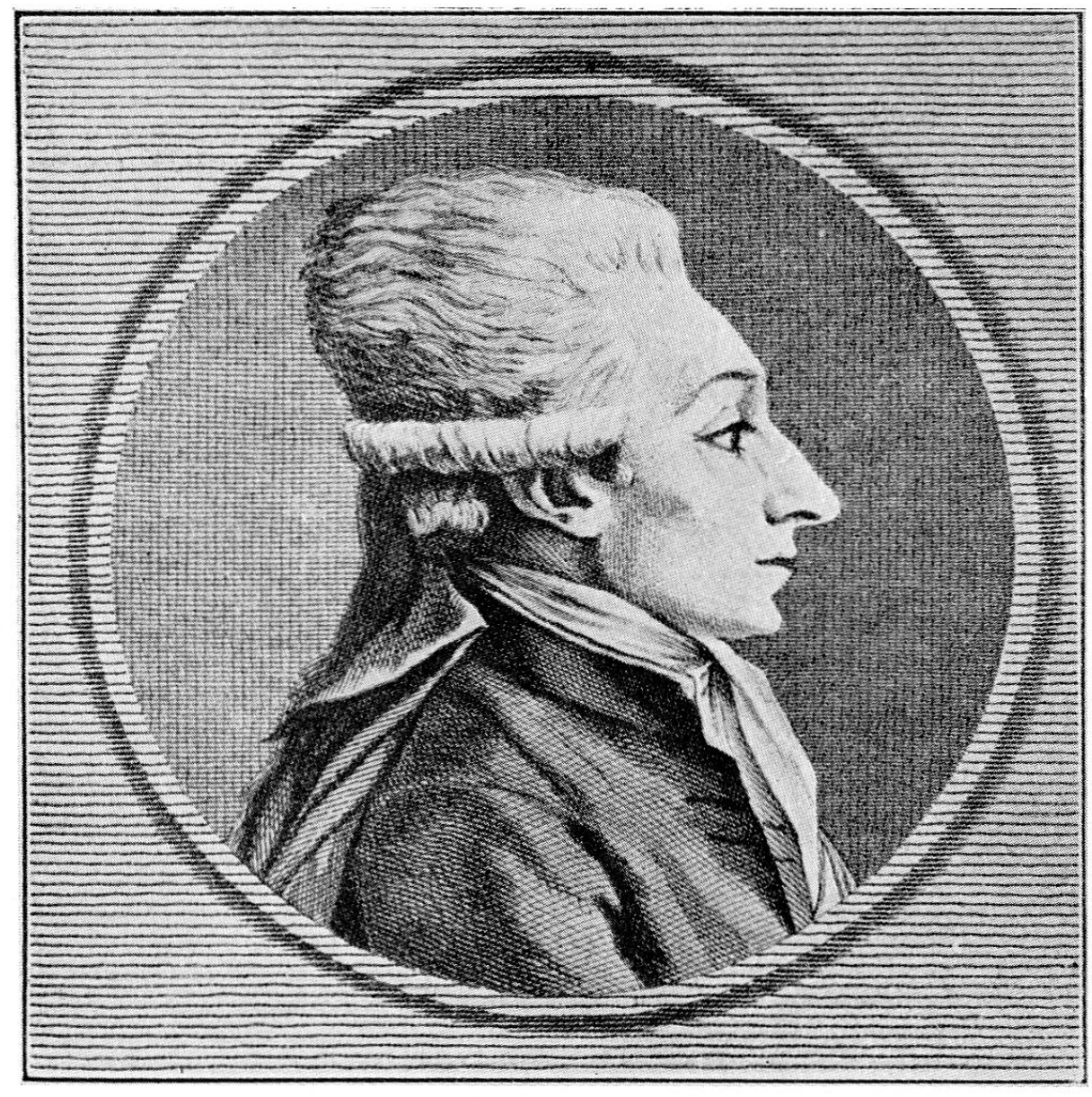

Maine de Biran (1766-1824). Photo Giraudon nach dem Original der Bibliothèque Nationale, Paris. Das von La Valette Monbrun (1914) und von Lemay (1936) veröffentlichte Porträt stellt nicht MaIne DE Biran, sondern Armand Louis de Gontaut, Duc de Biran dar 